\title{
Study on the pressure self-adaptive water-tight junction box in underwater vehicle
}

\author{
Haocai Huang ${ }^{1,2}$, Yanying Ye ${ }^{1}$, Jianxing Leng ${ }^{1,2}$, Zhuoli Yuan ${ }^{1}$ and Ying Chen ${ }^{1,2}$ \\ ${ }^{1}$ Institute of Underwater Technology and Ship Engineering, Zhejiang University, Hangzhou, China \\ ${ }^{2}$ State Key Lab of Fluid Power Transmission and Control, Zhejiang University, Hangzhou, China
}

\begin{abstract}
Underwater vehicles play a very important role in underwater engineering. Water-tight junction box (WJB) is one of the key components in underwater vehicle. This paper puts forward a pressure self-adaptive water-tight junction box (PSAWJB) which improves the reliability of the WJB significantly by solving the sealing and pressure problems in conventional WJB design. By redundancy design method, the pressure self-adaptive equalizer (PSAE) is designed in such a way that it consists of a piston pressure-adaptive compensator (PPAC) and a titanium film pressureadaptive compensator (TFPAC). According to hydro-mechanical simulations, the operating volume of the PSAE is more than or equal to $11.6 \%$ of the volume of WJB liquid system. Furthermore, the required operating volume of the PSAE also increases as the gas content of oil, hydrostatic pressure or temperature difference increases. The reliability of the PSAWJB is proved by hyperbaric chamber tests.
\end{abstract}

KEY WORDS: Underwater vehicle; Pressure self-adaptive equalizer; Water-tight junction box; Redundancy design.

\section{INTRODUCTION}

Ocean covers about 71 percent of the earth's surface and contains abundant resources. The development of ocean resources can be the final solution to some global science and engineering issues such as the study of origin of life, development of new resources, the improvement of human living condition, social sustainable development and so on (Jiang, 2000; Chen, Yang, Gu and Ye, 2003; Wang, Yuan, Li and Jiao, 2011; Nihous, 2007; Izadparast and Niedzwecki, 2011). However, the underwater environment (e.g., high pressure) makes underwater resource exploration quite challenging, especially in deep sea. Nowadays, with the development of marine technology, a variety of underwater vehicles have been developed and are playing an important role in underwater resource exploration, inspection and operation (Li, et al., 2009; Bessa, Dutra and kreuzer, 2010; Fang, Hou and Luo, 2007).

Underwater vehicles use multiple sensors, working components and control systems to achieve precise movement and accomplish certain tasks. Water-tight junction boxes (WJB) are used to protect the connections between sensors and working components from water and ensure the reliability of underwater vehicles (Chyba, Haberkorn, Smith and Choi, 2008; Bagheri, Karimi and Amanifard, 2010). As the water depth increases, WJBs suffer from increasing external water pressure. If the WJB uses pressure hull design, the structure of the WJB will be simple but the size and weight of the WJB will be increased significantly. The cumbersome WJB will occupy a large space, thus increasing the weight of the underwater vehicle and degrading the power efficiency, maintainability and reliability of the underwater vehicle (Zhang, Luo, Wang and Gu, 2007). From the other hand, if the WJB is connected with an external pressure compensator, then the internal and external pressure can be

Corresponding author: Jianxing Leng

e-mail: jxleng@zju.edu.cn 
balanced; however, some hidden dangers occur, e.g., pipe blockage, leakage, corrosion and damage of external compensator.

This paper puts forward a pressure self-adaptive water-tight junction box (PSAWJB) using a piston pressure-adaptive compensator (PPAC) and a titanium film pressure-adaptive compensator (TFPAC), which makes the pressure shell more lightweight. The pressure self-adaptive equalizer (PSAE) doesn't need any external compensator or oil line. Therefore the complexity of the structure is reduced and some hidden dangers (e.g., pipe blockage, leakage, etc.) are avoided. The PPAC makes the internal pressure always a bit higher than the external water pressure such that external water is prevented from leaking into the oil chamber. Titanium has large deformation range and excellent aging resistance, therefore selected for the film of the pressure-adaptive compensator. By this two-compensator design, the reliability of the WJB is improved significantly.

\section{DESIGN OF PSAWJB}

"Pressure self-adaptive" means that it can automatically change its pressure according to the external pressure. There are many types of pressure self-adaptive compensators, such as metal film type, bag type, corrugated pipe type and piston type of pressure compensator (Cao, et al., 2011; Meng, Wang, Wei and Zhang, 2000). The working principles of the metal film type, bag type and corrugated pipe type of pressure compensators are similar, which are commonly featured as a thin-wall vessel that allows a certain degree of elastic deformation (Meng, Wang, Wei and Zhang, 2000). The PPAC is designed using non-elastic shell. Pressure compensation is achieved by volume change in the piston chamber (Zhang, Luo, Wang and Gu, 2007). Consider the limited space in medium sized work-class underwater vehicles in deep-sea high pressure environment, these pressure compensators have certain disadvantage. Metal film, bag and corrugated pipe types are pressed by seawater to cause elastic deformation. The pressure of the external water is always higher than the internal oil during deformation, such that, external water might leak into the oil chamber and lead to short-circuit in electronic devices. Materiel of most bag and corrugated pipe are rubber which ages easily in seawater. Although corrugated pipe type can be made of Titanium, it is difficult in processing. Deep-sea environment need large volume of compensation which lead to a large and heavy piston. Pressure compensators' characteristics are summarized in Table 1.

Table 1 Characteristics of several types of pressure compensators.

\begin{tabular}{|c|c|c|c|c|c|c|}
\hline Characteristics & Metal film & Bag & $\begin{array}{c}\text { Corrugated pipe } \\
\text { (rubber) }\end{array}$ & $\begin{array}{c}\text { Corrugated pipe } \\
\text { (Titanium) }\end{array}$ & Piston & PSAWJB \\
\hline Non-leakage & $\times$ & $\times$ & $\times$ & $\times$ & $\sqrt{ }$ & $\sqrt{ }$ \\
\hline Anti-aging & $\sqrt{ }$ & $\times$ & $\times$ & $\sqrt{ }$ & $\sqrt{ }$ \\
\hline Compact structure & $\sqrt{ }$ & $\sqrt{ }$ & $\sqrt{ }$ & $\sqrt{ }$ & $\times$ & $\sqrt{ }$ \\
\hline Light Weight & $\sqrt{ }$ & $\sqrt{ }$ & $\sqrt{ }$ & $\sqrt{ }$ & $\times$ & $\sqrt{ }$ \\
\hline Easy process ability & $\sqrt{ }$ & $\sqrt{ }$ & & $\checkmark$ \\
\hline
\end{tabular}

Considering the characteristics of several types of pressure compensators in Table 1, PSAWJB is put forward in this paper. The PSAWJB has the following merits: a PPAC is used for pressure compensation which makes the internal pressure always a bit higher than the external pressure, thus preventing external water from infiltrating the oil chamber. Using the redundancy design method, a TFPAC is used as volume compensation, making the PPAC design more compact and more reliable. Titanium of the TFPAC is resistant to seawater corrosion and aging, thus improving the lifetime of the structure. By this design, the PASWJB can be an appropriate pressure self-adaptive system in deep-sea environment.

\section{The overall design}

Schematic of the PSAWJB in underwater vehicle presented in this paper is shown in Fig. 1. Fig. 1(a) is the explosion view, showing the structure of the PSAWJB and its main components. Fig. 1(b) is the wire frame showing the dimensions of the PSAWJB (400 $\mathrm{mm}$ in length, $230 \mathrm{~mm}$ in width and $278 \mathrm{~mm}$ in height). The PSAWJB in this paper is fitted with medium sized work-class underwater vehicles. 


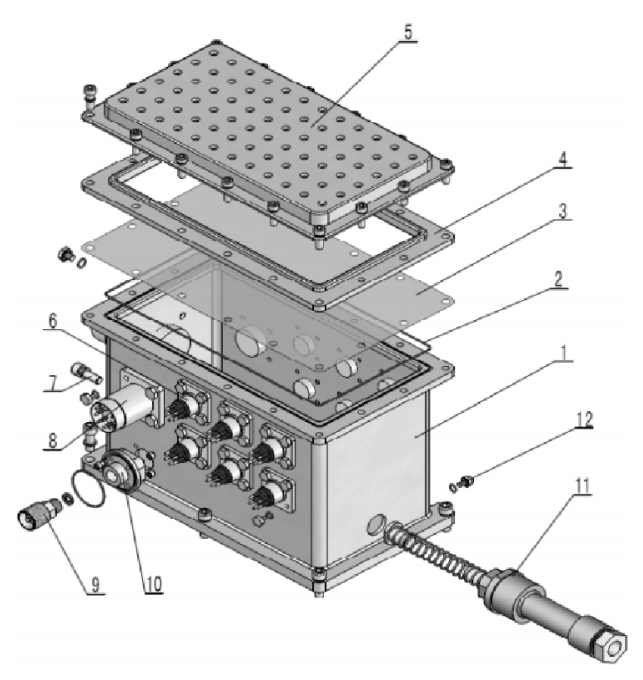

(a)

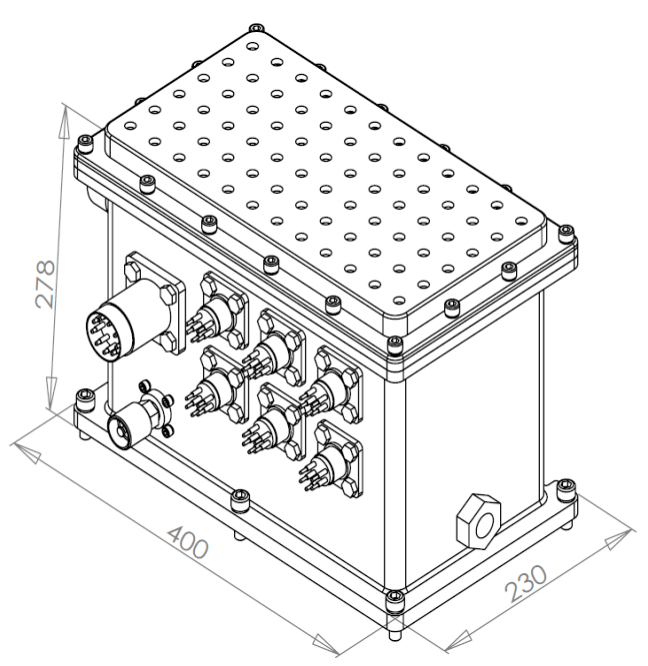

(b)

Fig. 1 The pressure self-adaptive water-tight junction box in underwater vehicle.

The specific compositions of the PSAWJB are shown in Fig. 1(a). The PSAWJB includes main components as follows: 1box, 2-sealing strip, 3-titanium film, 4-pressure plate, 5-hood, 6,8-underwater electrical connector, 7-plug, 9-quick coupler, 10quick coupler fixing pedestal, 11-PPAC, 12-oil entrance and exit pore. Underwater electrical connectors are installed on the wall of the box. In order to ensure the sealing performance, each mounting hole has two axial and radial O-rings. The PPAC is installed at the bottom of the wall of the box. The TFPAC is installed on the top of the box by the pressure plate which sealed by the sealing strip. The hood is installed on the pressure plate to prevent the outside object damaging the titanium film.

\section{The working principle}

The PSAWJB connects multiple sensors and working components in underwater vehicles. As shown in Fig. 2, core sensors such as Depth, Attitude and Heading Reference System (AHRS) and Doppler Velocity Log (DVL), lights, cameras, valve systems and other optional equipment sensors are all connected in the PSAWJB.

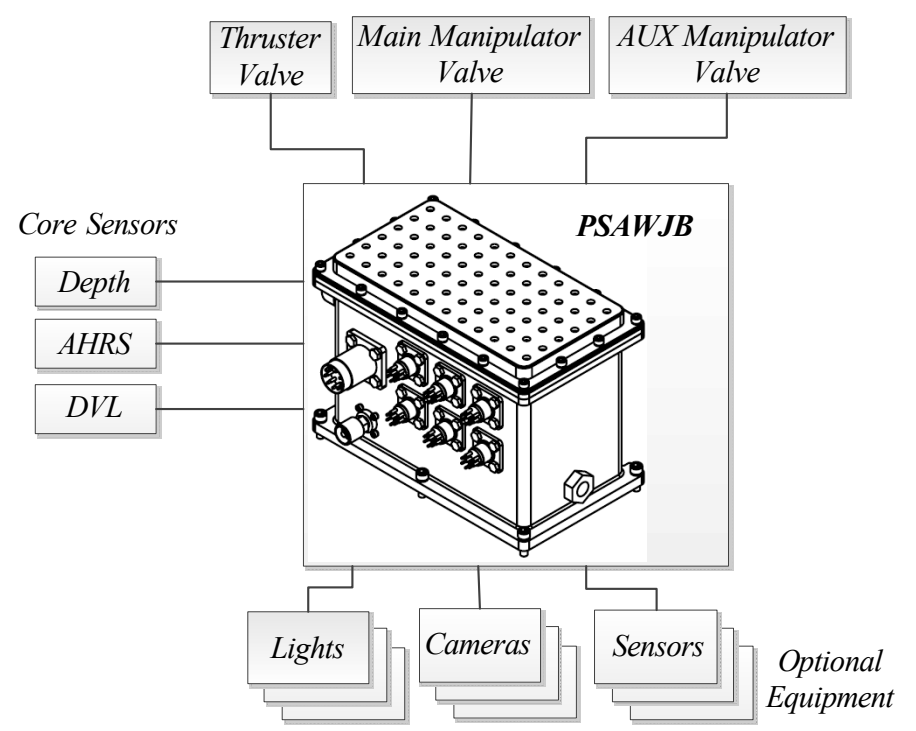

Fig. 2 Connection between sensors, PSAWJB and working components in underwater vehicles.

Firstly, components to the WJB and electrical wirings inside the WJB are all connected in air. The PPAC is installed on the 
junction box wall. Oil is filled from the oil entrance and gas is exhausted via the exit pore. The exit pore is plugged when the PSAWJB is full of oil and no gas remains. Keep on filling oil until the pressure reach $0.05 \mathrm{MPa}$. At this time the titanium film bulges outward.

As the underwater vehicle dives, the external pressure of the WJB increases. On one hand, the PPAC is pushed by water. The piston of the PPAC moves inside and the volume of the oil chamber is reduced. The oil is pressed and the oil pressure increases. On the other hand, the titanium film and the oil make up a built-in pressure compensator. The titanium film is pressed by water, the oil volume is reduced and the oil pressure increases. As the underwater vehicle rises, the piston and the titanium film are pressed by the oil. The volume of the oil chamber increases and the oil pressure reduces. In this way, the WJB is able to balance the internal and external pressure by the PPAC and TFPAC automatically. In addition, a pressure spring is installed at the end of the piston. The pressure of the oil chamber is the sum of the pressure of external water and the pressure spring, making the internal oil pressure always a bit higher than the external water pressure. This, pressure difference prevents external water from infiltrating the oil chamber. Built-in pressure compensators can also avoid the hidden dangers of using external pressure compensators.

\section{STUDY OF THE PSAE IN THE PSAWJB}

\section{Calculation of the compensator operating volume}

As different from pressure compensators operated in air, when the operating volume of a deep sea pressure compensator is calculated, the additional volume change of the oil in the oil-filled chamber caused by the pressure change should be considered (Zhang, Luo, Wang and Gu, 2007; Huang, et al., 2010):

$$
\begin{aligned}
& \Delta V_{Y(Y)}=\beta V_{Y} P_{J} \\
& \beta=\frac{a+2 b P_{J}}{1+a P_{J}+b P_{J}^{2}}
\end{aligned}
$$

In Eq. 1 and Eq. 2, $\Delta V_{Y(Y)}$ is the volume change of pure working liquid caused by pressure change. $\beta$ represents the comparative volume compressibility. $V_{Y}$ is the volume of the liquid system, where the unit of $V_{Y}$ is $m^{3} . P_{J}$ is the maximum hydrostatic pressure whose unit is MPa. Coefficients $a$ and $b$ are empirical coefficients. As for mineral oil, $a=6.13 \times 10^{-4}, b=1115 \times 10^{-9}$.

When the WJB is sent into deep-sea, the air volume change caused by the pressure change $\Delta V_{Q(Y)}$ can be almost neglected (Zhang, Luo, Wang and Gu, 2007), namely $\Delta V_{Q(Y)}=0$. A model of the operating volume of the PSAE $V_{B}$ in $\mathrm{m}^{3}$ is built according to hydromechanics (Zhang, Luo, Wang and Gu, 2007; Meng, Wang, Wei and Zhang, 2000; Huang, et al., 2010; He, Meng and Zhao, 2007; Munson, Young and Theofore, 2010).

$$
V_{B} \geq \Delta V_{Y(Y)}+\Delta V_{Y(W)}+\Delta V_{Q(W)}+\Delta V_{L}+\Delta V_{G}+\Delta V_{B G}
$$

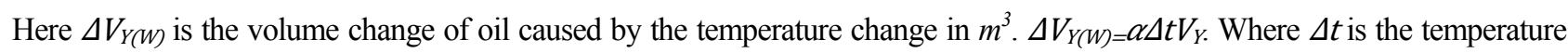
change in ${ }^{\circ} \mathrm{C}$. $\alpha$ is the volume expansion temperature coefficient. As for general mineral oil, $\alpha=5.8 \times 10^{-4} \sim 9.5 \times 10^{-4} . \Delta V_{Q(W)}$ is the gas volume change caused by temperature change in $m^{3} . \Delta V_{Q(W)}=V_{Y} K_{X} . K_{X}$ is the gas coefficient which can be taken as $3 \sim 6 \%$ for normal oil. In this paper, $K_{X}$ is set as $6 \% . \Delta V_{L}$ is the oil leakage volume in 48 hours in $m^{3} . \Delta V_{G}$ is the volume that the whole system needs to compensate (such as the volume change of the cylinder of single piston rod) in $m^{3}$ (Zhang, Luo, Wang and Gu, 2007). $\Delta V_{B G}$ is the change of the compensator operating volume caused by the change of pressure and temperature in $m^{3}$. Under the uniform liquid condition in the pressure compensator liquid system, $\Delta V_{B G}$ could be (Zhang, Luo, Wang and Gu, 2007; Meng, Wang, Wei and Zhang, 2000; Huang, et al., 2010): 


$$
\Delta V_{B G}=V_{B} C=V_{B} \sum \Delta V / V_{Y}
$$

$C$ is the compensator volume change coefficient. $\sum \Delta V$ is the volume of the operating liquid for the compensatory system.

$$
\sum \Delta V=\Delta V_{Y(\mathrm{Y})}+\Delta V_{Y(\mathrm{w})}+\Delta V_{Q(\mathrm{w})}+\Delta V_{L}+\Delta V_{G}=V_{Y}\left(\beta P_{J}+\alpha \Delta \mathrm{t}+K_{X}\right)+\Delta V_{L}+\Delta V_{G}
$$

In order to simplify the design, the effect of $\Delta V_{L}$ and $\Delta V_{G}$ is neglected. According to Eq. 4 and Eq. 5, we have:

$$
\begin{gathered}
\sum \Delta V=V_{Y}\left(\beta P_{J}+\alpha \Delta \mathrm{t}+K_{X}\right) \\
C=\left(\beta P_{J}+\alpha \Delta t+K_{X}\right)
\end{gathered}
$$

So the final expression of $V_{B}$ can be:

$$
V_{B} \geq \frac{C}{1-\mathrm{C}} V_{Y}=\frac{\beta P_{J}+\alpha \Delta \mathrm{t}+K_{X}}{1-\left(\beta P_{J}+\alpha \Delta \mathrm{t}+K_{X}\right)} V_{Y}
$$

According to the specified design requirements, the total volume of the WJB is about $10 L . V_{Y}$ is about $9 L$ considering the volume of components installed inside the WJB. Take 4,000 $\mathrm{m}$ water depth as an example, $P_{J}$ can be taken as $40 \mathrm{MPa}$. The temperature under water is about $4{ }^{\circ} \mathrm{C}$. The temperature on board is about $30^{\circ} \mathrm{C}$. Take $\alpha$ as $6 \times 10^{-4}$. According to Eq. 2, $\beta=7.00 \times 10^{-4}$. According to Eq. $7, C=0.054$. According to Eq. 8, we have:

$$
V_{B} \geq 11.6 \% V_{Y}
$$

Put $V_{Y}$ into Eq. 9, we get $V_{B} \geq 1,044 \mathrm{~mL}$.

\section{Volume variation of pressure compensator}

The WJB needs to be filled with oil before operation. The thermal expansion coefficient of gas is larger than that of oil, therefore the gas content in oil has significant impact on the volume of the PSAE. Assuming that other conditions remain unchanged and only $K_{X}$ changes $\left(P_{J}=40 \mathrm{MPa} . \Delta t=26^{\circ} \mathrm{C} . \alpha=6 \times 10^{-4}\right)$. According to Eq. 2 and Eq. 8 , the relationship between $K_{X}$ and $V_{B}$ is investigated (see Fig. 3).

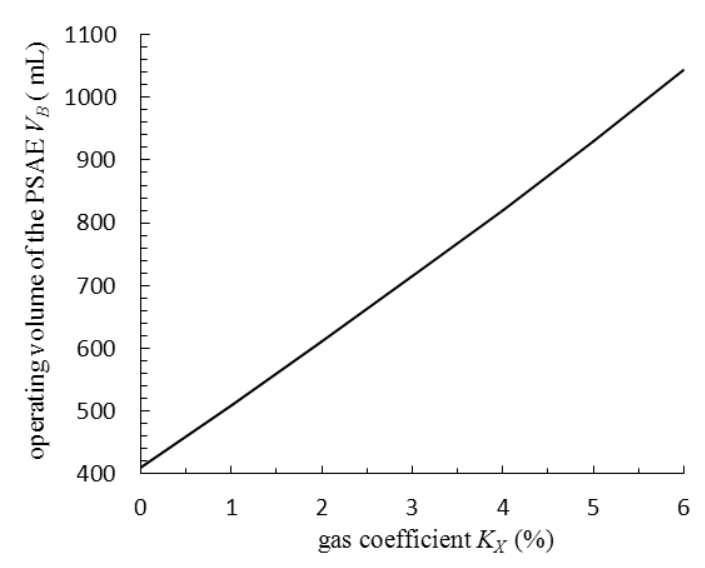

Fig. 3 The relationship between $K_{X}$ and $V_{B}$.

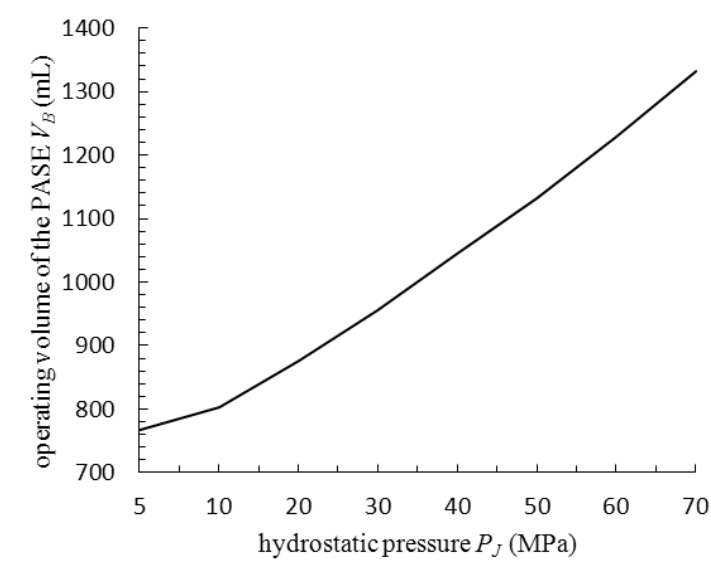

Fig. 4 The relationship between $P_{J}$ and $V_{B}$. 
Under the condition that temperature difference $\Delta t$ stays unchanged $\left(K_{X}=0.06, \Delta t=26^{\circ} \mathrm{C}, \alpha=6 \times 10^{-4}\right)$, relationship between $P_{J}$ and $V_{B}$ is investigated according to Eq. 2 and Eq. 8. The result is shown in Fig. 4. As the operating depth stays unchanged $\left(P_{J}=40 \mathrm{MPa}, K_{X}=0.06, \alpha=6 \times 10^{-4}\right)$, the relationship between $\Delta t$ and $V_{B}$ is investigated according to Eq. 2 and Eq. 8 (see Fig. 5).

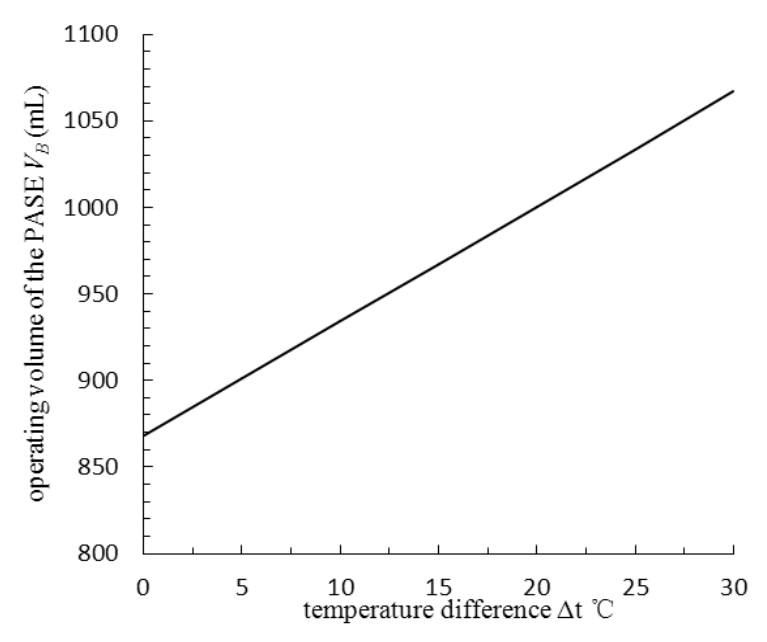

Fig. 5 The relationship between $\Delta t$ and $V_{B}$.

From Fig. 3, Fig. 4 and Fig. 5, we can clearly see that the operating volume $\left(V_{B}\right)$ of the PSAE increases with the gas coefficient of the oil, the hydrostatic pressure and the temperature difference. Thus, degassing process of the oil is important for PSAWJB if it need to work deeper. It is better if the sea surface temperature is near the deep sea temperature.

\section{Study of the PPAC}

The structure of the PPAC is shown in Fig. 6. The piston rod of the PPAC is installed in the cylinder block. One side of the piston is connected with the internal oil chamber and the other side is connected with the external water environment. The piston is sealed by the sealing end cover to prevent the water from leaking into the internal oil chamber. The PPAC is fixed with the box by the nut. During underwater operation, the increasing pressure of the external water pushes the piston to slide, which makes the internal oil chamber volume reduced. Because hermetical fluid has a certain compressibility (including the compression of the residual gas in the oil), the pressure of the oil chamber will automatically balance the outer pressure according to

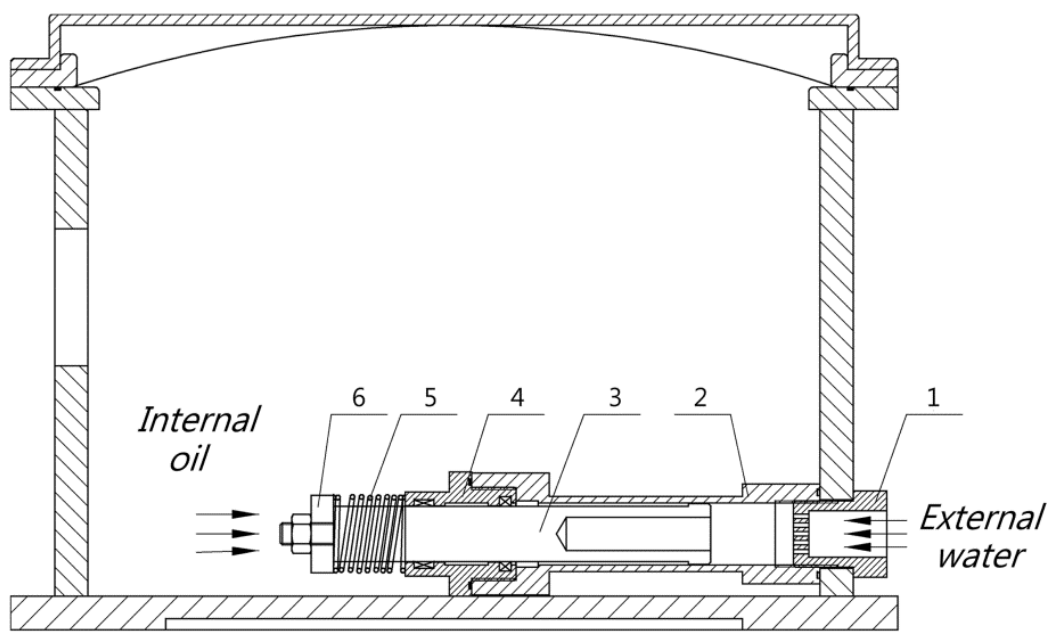

Fig. 6 Structure of the PPAC.

1-nut, 2-cylinder block, 3-piston rod, 4-sealing end cover, 5-pressure spring, 6-spring nut. 
Pascal's Law. A pressure spring is installed on the piston rod by the spring nut. The pressure of the oil chamber is the sum of the pressure of external water and the pressure spring, making the internal oil pressure always higher than the external water pressure. In this condition, it could prevent the external water from infiltrating into the oil chamber (the hyperbaric chamber test proved it is no leakage problem of the oil). Thus it won't cause short circuit in the PSAWJB. Furthermore, thread can be machined on the piston by mechanical design which makes the pressure of the spring adjustable. As a result, the pressure difference of the internal of the WJB and external water environment can be adjusted which makes the compression resistance of the WJB increases.

When the PPAC is working, the internal static force balance equation is:

$$
P_{C} \cdot A=P_{E} \cdot A+F_{K}
$$

Here $P_{C}$ is pressure of oil inside the PPAC. $P_{E}$ is the pressure of external water. $F_{K}$ is the spring force. $A$ is the piston area of the PPAC.

Given $k$ the elasticity coefficient of the pressure spring in the PPAC, $x$ the compressed volume of the pressure spring, $F_{K}$ can be written as (according to Hooke's law):

$$
F_{K}=k \cdot x
$$

From Eq. 10 and Eq. 11, the internal and external pressure difference $\Delta P$ is calculated as (Zhang, Luo, Wang and Gu, 2007):

$$
\Delta P=P_{C}-P_{E}=\frac{F_{K}}{A}=\frac{k \cdot x}{A}
$$

Eq. 12 shows that the internal pressure of the PPAC is always higher than the external pressure because of the spring force, despite the water depth and the external water pressure.

By specific design of the piston, screw rod can be machined where the spring is installed. As a result, the preload of the spring is adjustable, which makes $\Delta P$ can be adapted for difference water depth. Piston diameter is $25 \mathrm{~mm}$. The stiffness coefficient $k$ is $10 \mathrm{~N} / \mathrm{mm}$. The relationship between $\Delta P$ and adjustable length of the spring $x$ is shown in Fig. 7. From Fig. 7 we can see that the longer the $x$, the larger the $\Delta P$.

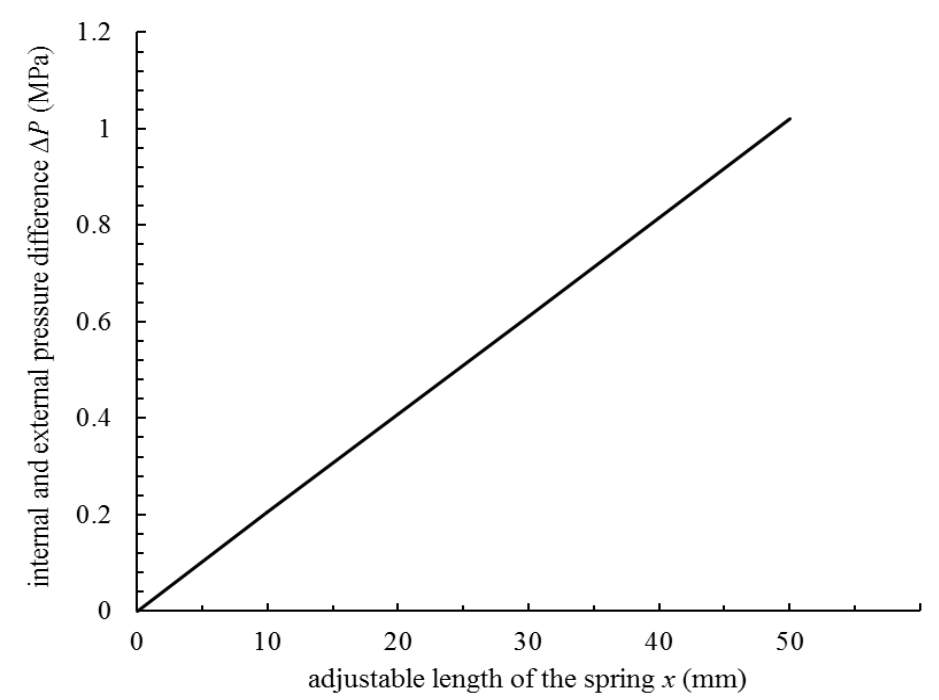

Fig. 7 The relationship between $\Delta P$ and $x$. 


\section{Study of the TFPAC}

Considering redundancy design and volume compensation, a volume compensator for the WJB is designed. According to Table 1, bag and corrugated pipe type compensators have aging and leakage problems. Therefore we use the metal film pressure compensator and the material of the film is titanium. The corrosion and ageing resistance of titanium is excellent, making it work well in seawater environment. The extensibility of titanium film is very good, therefore the film can be made large enough to meet the requirements of deep-sea pressure compensation. The TFPAC is filled with oil and directly placed in water. When the titanium film is pressed by water, it will produce elastic deformation which reduces the volume, making the pressure of the internal oil increases. So, no matter how deep the water is, the TFPAC makes the internal oil pressure equal to the external water pressure.

In this paper, the TFPAC is composed of box, titanium film, sealing strip, pressure plate and shield (see Fig. 8). The thickness of titanium film is only $0.1 \mathrm{~mm}$. The pressure plate is used to ensure the sealing performance of the sealing strip. The box is filled with pre-pressured oil, making the titanium film show a protruding status (see Fig. 8(a)). During underwater operation, the external water pressure of the TFPAC increases. Due to the elastic deformation of the titanium film, the volume of the oil inside the WJB is reduced and the oil pressure is increased (see Fig. 8(b)). As a result, the WJB can adapt to different water pressure and eliminate the effects of water pressure.

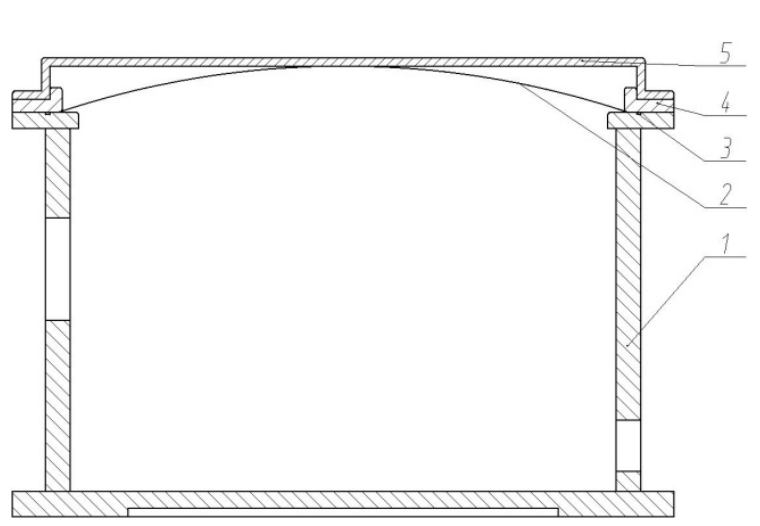

(a)

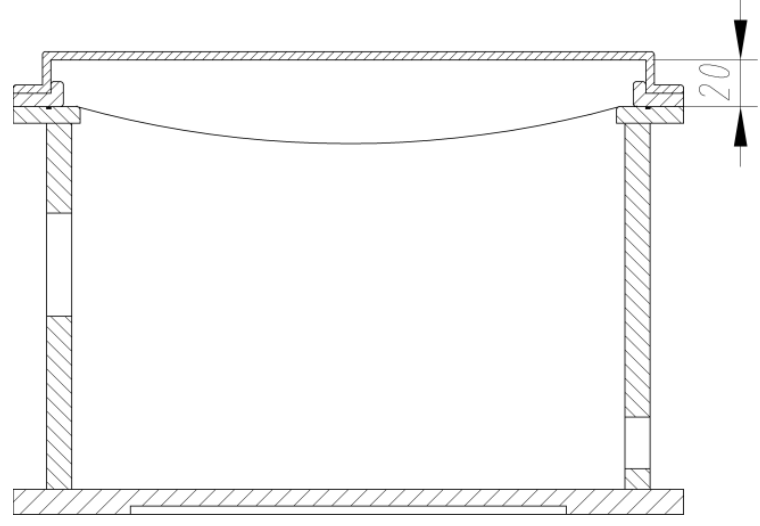

(b)

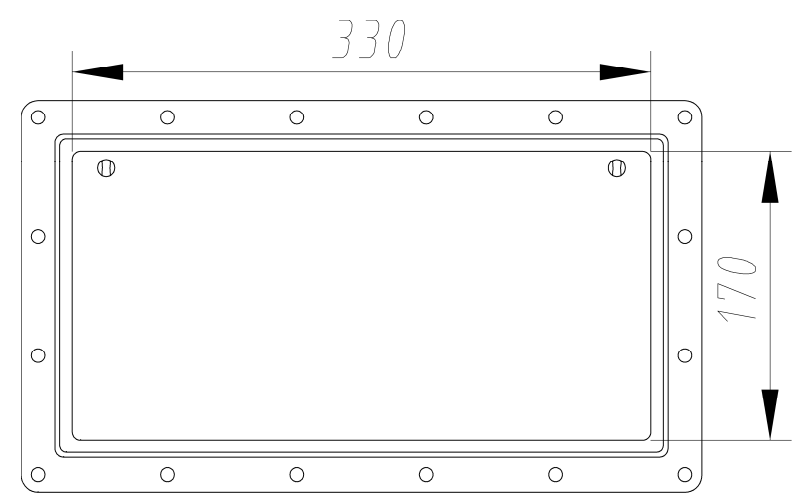

(c)

Fig. 8 Schematic diagram of the TFPAC.

1-box, 2-titanium film, 3-sealing strip, 4-pressure plate, 5-shield.

Titanium film has good extensibility making it suitable to be the volume compensator. The expansion size of the titanium film is $170 \times 330 \mathrm{~mm}$ (see Fig. 8(c)). The height of the shield is $20 \mathrm{~mm}$ (see Fig. 8(b)). Considering the critical case, the titanium film can be expanded to fill the gap of the shield. When the titanium film is pressed by water, the compression volume can be considered equal to the expansion volume. The largest compensation volume is $V_{\max }=17 \times 33 \times 2 \times 2=2,244 \mathrm{~mL}$. The operating volume of the compensator is $V_{B} \geq 11.6 \% V_{B}=1,044 \mathrm{~mL}$. $V_{\max }$ is larger than $V_{B}$. According to Fig. $3, V_{B}$ is even larger than the compensation volume when the pressure is $70 M P a$, that is, the WJB can be operated properly in 7,000 $\mathrm{m}$ depth. 
By combining the TFPAC and PPAC, the operating volume of PPAC can be reduced. This makes PSAWJB compact and light-weighted. The reliability of the PSAWJB is also improved.

\section{HYPERBARIC CHAMBER TESTS}

The PSAWJB has been tested in the hyperbaric chamber to prove its feasibility and reliability. The tests have been carried out in the State Key Lab of Fluid Power Transmission and Control. As shown in the Fig. 9(a), the highest pressure of the hyperbaric chamber can be adjusted up to $60 \mathrm{MPa}$. In order to test the feasibility and reliability of the PPAC, pressure impact test is needed. To prove the pressure resistance of the PSAWJB, pressure keeping test is needed.

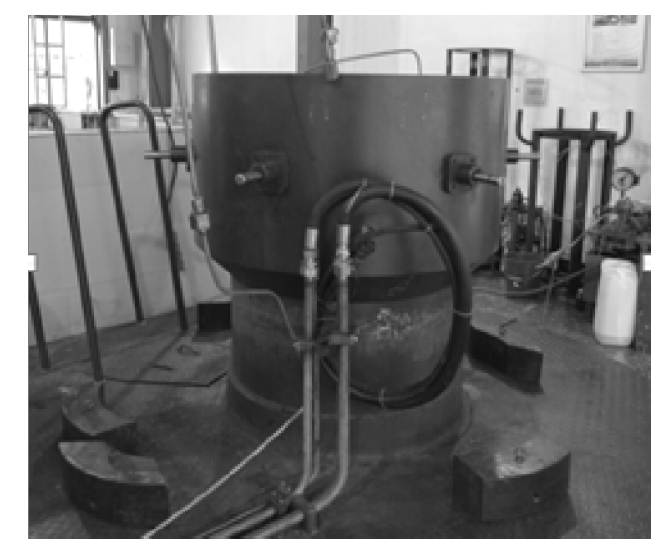

(a)

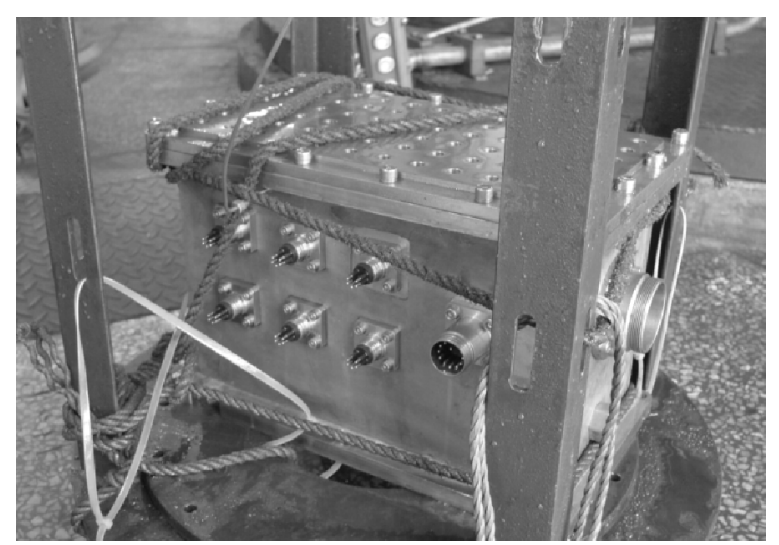

(b)

Fig. 9 Hyperbaric chamber test.

The pressure impact test is carried out as follows:

(1) Check the seal of the PSAWJB. Fill the hydraulic oil into it and completely evacuate the gas inside the PSAWJB.

(2) Fix the PSAWJB into the bracket, as shown in Fig. 9(b). Fill certain amount of water into the chamber. Hang the bracket into the chamber, close the lid and fix the plate, ensuring the seal of the hyperbaric chamber.

(3) Open the valves and switch on the pumps, continuously pump the water into the chamber, and evacuate the air completely.

(4) Close the valve, pump water into the hyperbaric chamber, making the water pressure reach the required pressure. Then switch off the pump.

(5) Keep up the pressure for some time. Then open the relief valve to relief the pressure to $0 \mathrm{MPa}$.

(6) Switch on the pump and continue to pump the water, evacuate the rest air in the hyperbaric chamber.

(7) Repeat steps (4) (6).

The pressure keeping test is carried out as follows:

(1) Check the seal of the PSAWJB. Fill the hydraulic oil into the PSAWJB such that the gas in the PSAWJB is completely evacuated.

(2) Fix the PSAWJB into the bracket, as shown in Fig. 9(b). Fill certain amount of water into the chamber. Hang the bracket into the chamber, close the lid and fix the plate, ensuring the seal of the hyperbaric chamber.

(3) Open the valves and switch on the pumps, continuously pump the water into the chamber, and evacuate the air completely.

(4) Close the valve, pump water into the hyperbaric chamber, making the water pressure reach the required pressure. Then switch off the pump.

(5) Keep up the pressure for 48 hours. Then open the relief valve to relief the pressure to $0 \mathrm{MPa}$.

In the pressure impact tests, the piston reciprocates so that the leakage in the PPAC can be tested. The 48 hours pressure keeping test can prove the reliability of the PSAWJB. Before the pressure impact test and pressure keeping test, the total 
weight of PSAWJB with oil is $63,310 \mathrm{~g}$. After tests, the weight of the PSAWJB remains to be $63,310 \mathrm{~g}$, which indicates that there's no leakage of the oil from the PSAWJB. The feasibility and reliability of the PSAWJB is proved through the hyperbaric chamber tests.

\section{CONCLUSIONS}

In this paper, a pressure self-adaptive water-tight junction box (PSAWJB) in underwater vehicle has been designed and tested. Main conclusions are drawn as follows:

(1) Redundancy design method is adopted to design a PSAWJB, which consists of a PPAC and a TFPAC. The PSAWJB is designed in such a way that the pressure inside and outside of the PSAWJB can be balanced automatically. Water leakage is prevented. The reliability of the PSAWJB is improved greatly.

(2) By modeling the volume variation of the PSAE in the PSAWJB, it's shown that the operating volume of the PSAE in 40 $M P a$ is more than or equal to $11.6 \%$ of the volume of PSAWJB liquid system. The necessary pressure compensating volume increases with the increase of the gas coefficient, temperature difference and the max hydrostatic pressure. Thus, degassing process of the oil is important for PSAWJB if it needs to work deeper.

(3) The feasibility and reliability of the PSAWJB is proved through hyperbaric chamber tests. The PSAWJB can operate properly in 7,000 $m$ deep-sea.

\section{ACKNOWLEDGMENT}

This work is financially supported by National High Technology Research and Development Program of China (863 Program Grant No.2009AA09A20401-1), and the Zhejiang Province Public Technology Application Research Project (Grant No. 2010C31107 and No. 2011C23107).

\section{REFERENCES}

Bagheri, A., Karimi, T. and Amanifard, N., 2010. Tracking performance control of a cable communicated underwater vehicle using adaptive neural network controllers. Applied Soft Computing, 10(3), pp.908-918.

Bessa, W.M., Dutra, M.S. and Kreuzer, E., 2010. An adaptive fuzzy sliding mode controller for remotely operated underwater vehicles. Robotics and Autonomous Systems, 58(1), pp.16-26.

Cao, X.P., Zhang, C.H., Deng, B., XieQ. and Rong, Y.L., 2011. Research on pressure-compensated dynamic property of deep-sea oil reservoir systems. Ocean Technology, 30(1), pp.83-87.

Chen, Y., Yang, C.J., Gu, L.Y. and Ye, Y., 2003. DSV-specific tool-box for the deep-sea resources exploitation. Chinese Journal of Mechanical Engineering, 39(11), pp.38-42.

Chyba, M., Haberkorn, T., Smith, R.N. and Choi, S.K., 2008. Design and implementation of time efficient trajectories for autonomous underwater vehicles. Ocean Engineering, 35(1), pp.63-76.

Fang, M.C., Hou, C.S. and Luo, J.H., 2007. On the motions of the underwater remotely operated vehicle with the umbilical cable effect. Ocean Engineering, 34, pp.1275-1289.

He, J., Meng, Q.X. and Zhao, J., 2007. The design of hydraulic pressure system for underwater interfacing manipulator. Hydraulics Pneumatics \& Seals, 1, pp.22-24.

Huang, H.C., Yang, C.J., Yang, Q.H., Zhou, L.J., Wang, H. and Niu, W.D., 2010. Study of gas-tight deep-sea water sampling system based on pressure self-adaptive equalization. Journal of Mechanical Engineering, 46(12), pp.148-154.

Izadparast, A.H. and Niedzwecki, J.M., 2011. Estimating the potential of ocean wave power resources. Ocean Engineering, 38(1), pp.177-185.

Jiang, X.S., Feng, X.S. and Wang, D.T., 2000. Unmanned underwater vehicles. Shenyang: Liaoning Science and Technology Publishing House. 
Li, Y., Pang, Y.J., Chen, Y., Wan, L. and Zou, J., 2009. A hull-inspect ROV control system architecture. China Ocean Engineering, 23(4), pp.751-761.

Meng, Q.X., Wang, Z., Wei, H.X. and Zhang, L.X., 2000. Development of pressure compensator for deep water hydraulic power station. Ship Engineering, 2, pp.60-61.

Munson, B.R., Young, D.F., Theofore, H.O. and Wade, W. H., 2009. Fundamentals of fluid mechanics. 6th ed. USA: John Wiley \& Sons.

Nihous, G.C., 2007. An estimate of Atlantic Ocean thermal energy conversion (OTEC) resources. Ocean Engineering, 34 (17-18), pp.2210-2221.

Wang, S.J., Yuan, P., Li, D. and Jiao, Y.H., 2011. An over view of ocean renewable energy in China. Renewable and Sustainable Energy Reviews, 15(1), pp.91-111.

Zhang, Y., Luo, G.Sh., Wang, F. and Gu, L.Y., 2007. Deep-sea pressure adaptive compensation technique for underwater robots. Mechanical \& Electrical Engineering Magazine, 24(4), pp.10-13. 